\title{
Aortic valve repair for tri-leaflet aortic insufficiency associated with asymmetric aortic root aneurysms
}

\author{
George T. Stavridis ${ }^{1}$, Richard S. Downey ${ }^{2}$, Marc W. Gerdisch ${ }^{3}$, G. Chad Hughes ${ }^{4}$, Marek J. Jasinski ${ }^{5}$, \\ J. Scott Rankin ${ }^{6}$, Domenico Mazzitelli ${ }^{7}$, Diana Aicher ${ }^{8}$, Theodor Fischlein ${ }^{9}$, Yeong-Hoon Choi ${ }^{10}$, \\ Alberto Weber ${ }^{11}$, Ivar Friedrich ${ }^{12}$, Mattia Glauber ${ }^{13}$, Steffen Pfeiffer ${ }^{4}$, Antonio Miceli ${ }^{8}$, Ming-Sing Si ${ }^{14}$, \\ Tomislav Klokocovnik ${ }^{15}$, Lawrence M. Wei ${ }^{6}$, Marek A. Deja ${ }^{10}$, Vinay Badhwar ${ }^{6}$
}

${ }^{1}$ Department of Cardiac Surgery, Onassis Cardiac Surgery Center, Athens, Greece; ${ }^{2}$ University of Michigan, Muskegon, MI, USA; ${ }^{3}$ Department of Cardiothoracic Surgery, Franciscan Hospital, Indianapolis, IN, USA; ${ }^{4}$ Division of Thoracic Surgery, Duke University Medical Center, Durham, NC, USA; ${ }^{5}$ Department of Cardiothoracic Surgery, Wroclaw Medical University, Wroclaw, Poland; ${ }^{6}$ Department of Cardiovascular and Thoracic Surgery, West Virginia University Heart and Vascular Institute, Morgantown, WV, USA; ${ }^{7}$ Department of Cardiac Surgery, Klinikum Bogenhausen, Munich, Germany; ${ }^{8}$ Bad Bevensen, Germany; ${ }^{9}$ Department of Cardiovascular Surgery, Klinikum Nürnberg, Paracelsus Medical University, Nürnberg, Germany; ${ }^{10}$ Department of Cardiac and Thoracic Surgery, University of Köln, Köln, Germany; ${ }^{11}$ Department of Cardiac Surgery, University of Zurich, Zurich, Switzerland; ${ }^{12}$ Department of Cardiac and Thoracic Surgery, Krankenhaus der Barmherzigen Brüder Trier, Trier, Germany; ${ }^{13}$ Department of Cardiac Surgery, Istituto Clinico Sant'Ambrogio, Gruppo Ospedaliero San Donato, Milan, Italy; ${ }^{14}$ University of Michigan, Mott Children's Hospital, Ann Arbor, MI, USA; ${ }^{15}$ Department of Cardiac Surgery, University Medical Center Ljubljana, Ljubljana, Slovenia

Correspondence to: J. Scott Rankin, MD. West Virginia University Heart and Vascular Institute, 1 Medical Center Drive, West Virginia University, Morgantown, WV, USA. Email: jsrankinmd@cs.com.

Submitted Jan 26, 2019. Accepted for publication Apr 24, 2019.

doi: 10.21037/acs.2019.04.10

View this article at: http://dx.doi.org/10.21037/acs.2019.04.10

\section{Clinical vignette}

The patient was a 47 -year-old male with mild heart failure and an expanding $5.2-\mathrm{cm}$ ascending aortic aneurysm. His sinuses also were involved, with a $6.3-\mathrm{cm}$ aortic root diameter. He had Grade 4 aortic insufficiency (AI) (Figure 1) and his left ventricle and coronaries were normal. His aortic annulus was especially dilated at $30 \mathrm{~mm}$. The right and non-coronary sinuses were primarily involved in the root aneurysm and his left sinus seemed normal. Echocardiography showed the dilated annulus, leaflet tethering from the sinotubular junction (STJ) dilatation and normal appearing leaflets. The AI was central and severe with stretching of the leaflets and loss of central coaptation. No leaflet prolapses or other defects were evident.

\section{Surgical technique}

\section{Preparation-exposition}

In this patient, we chose to (I) repair the aortic valve and (II) to selectively replace only the aneurysmal right and non-coronary sinuses. Midline sternotomy was employed and conventional cardiopulmonary bypass via the distal ascending aorta and the right atrium was utilized, with anterograde and retrograde Custodiol ${ }^{\mathrm{TM}}$ cardioplegia. Initially, we applied retrograde cardioplegia to arrest the heart followed by direct administration into both coronary ostia.

\section{Operation}

The aorta was opened high, to avoid possibly displaced coronary ostia, and a circular aortotomy was completed down to within $1 \mathrm{~cm}$ of the coronaries and commissural tops. The ascending aneurysm was excised and again, the right and non-coronary sinuses appeared aneurysmal, with a normal left sinus and nice leaflets. Traction sutures were placed horizontally above each commissural top, and the annulus sized to $30 \mathrm{~mm}$ (video: http://www.jsrmd.com/ ftp/245_ARAc.mp4). The commissure-to-commissure freeedge length of the left coronary leaflet suggested a $23-\mathrm{mm}$ ring, as did the non-coronary; however, the right leaflet 

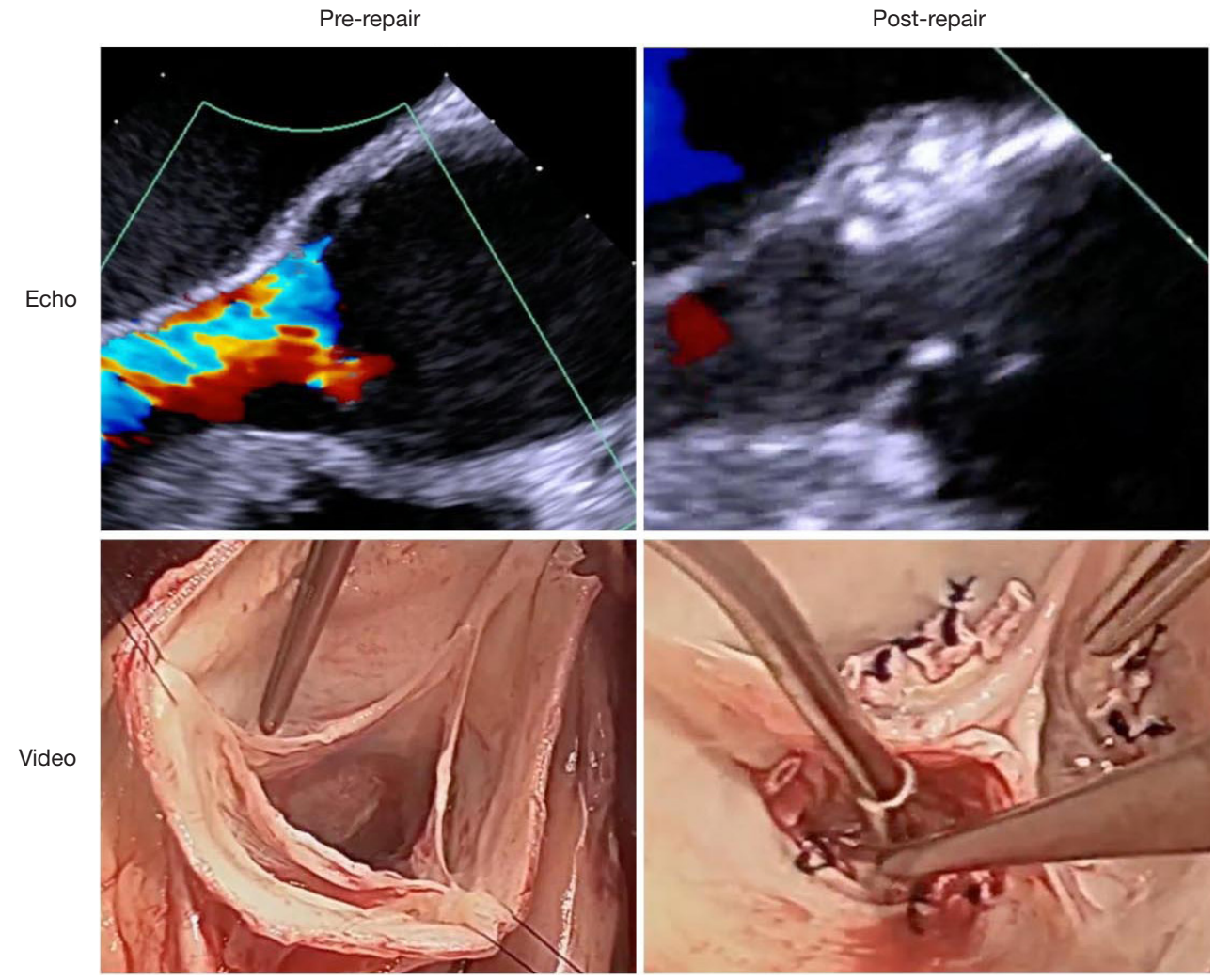

Figure 1 Echocardiographic and video images of the aortic valve, before and after the repair.

was 25 . Therefore, the ring was downsized to 23 , with the minor axis left/non-coronary post facing posteriorly.

\section{Aortic valve repair}

Positioning the ring posts low into the subcommissural triangles raises the tops of the commissures relative to the valve base and increases vertical leaflet coaptation height. To accomplish this goal, each subcommissural triangle was marked with a blue dot, $3 \mathrm{~mm}$ below the top of the subcommissural space (video: http://www.jsrmd.com/ ftp/245_ARAc.mp4). During subsequent ring placement, all commissural annular sutures were kept at or below the blue dot. With the ring above the valve, each commissural post was sutured into the subcommissural triangle using Cabrollike 4-0 Prolene horizontal mattress sutures. The ring was lowered below the valve, and two looping annular sutures were placed in each sinus-effectively looping the ring. For a \#19 annuloplasty ring, only one looping suture per sinus would be required (6 total sutures). Small supra-annular Dacron pledgets were positioned on each suture.

After all annular sutures were placed, the three commissural sutures were tied tightly as a first step with eight good knots. Getting the sutures tied tightly is especially important. Then, one needle was passed downward and lateral through the pledget (away from the leaflet) and tied with 6-7 more knots. This "lateral suture fixation" has minimized technical complications, such as knot untying and leaflet contact with the suture tails. At the end, all suture knot towers faced laterally and the ring was buried into the annulus and could not be seen. Leaflet coaptation was tested by "pressurizing" all three leaflets simultaneously with closed DeBakey forceps and the leaflets were quite vertical, coapted well in the midline and had equivalent effective heights.

\section{Aortic root replacement}

Attention then was turned to the aorta. Normal segmental sinus circumference should be just a little larger than the leaflet free-edge length (1-3) and in this patient, the right sinus was almost twice that dimension (see video). The noncoronary sinus also was much larger, but the left sinus was normal. The non-coronary and right sinuses were excised 

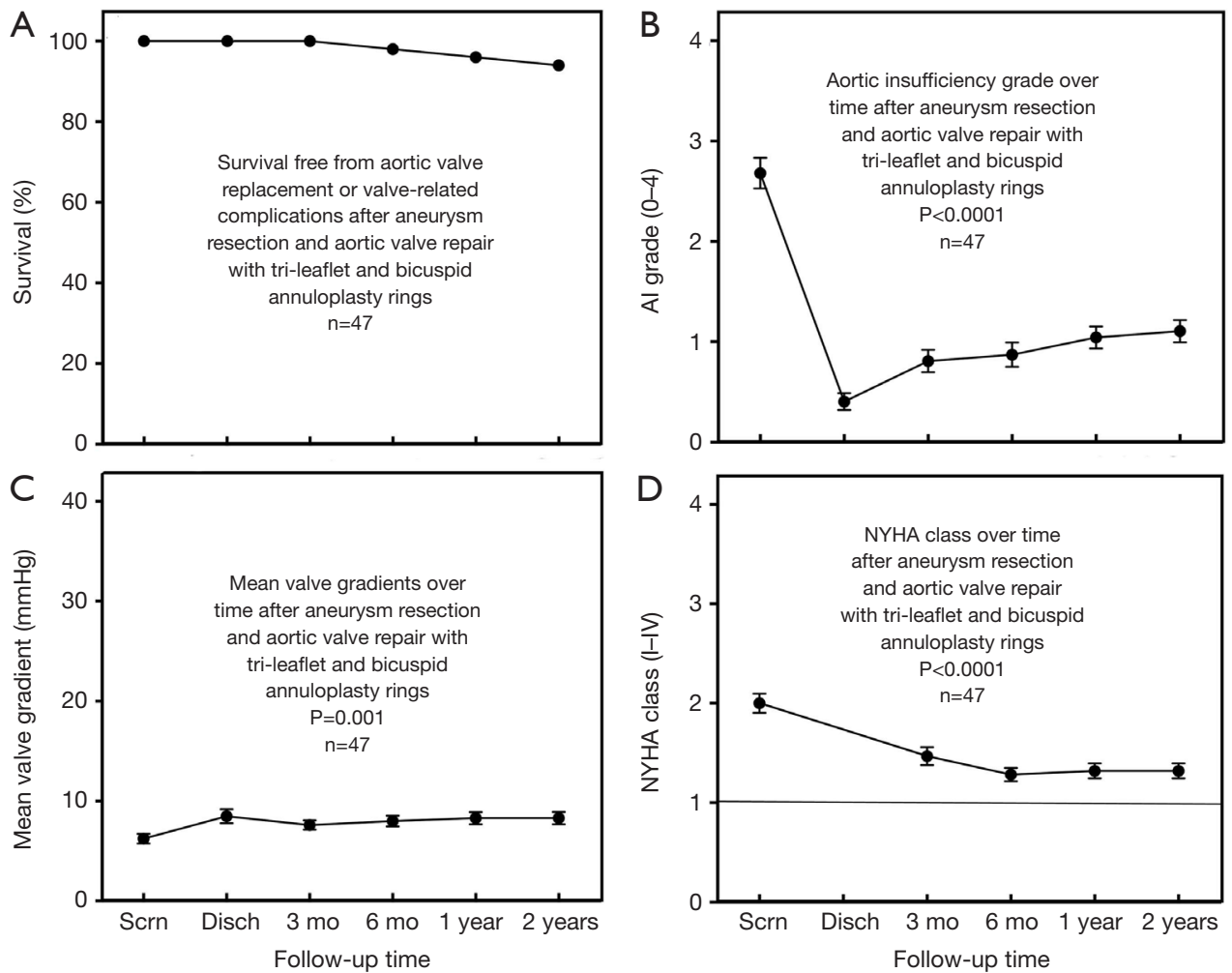

Figure 2 Outcome data after aortic valve repair associated with aortic aneurysm surgery using geometric ring annuloplasty. Scrn, screening; Disch, discharge; mo, month; NYHA, New York Heart Association.

and the right coronary button was developed. A 30-mm Valsalva graft (7 $\mathrm{mm}$ larger than the annuloplasty ring) was chosen and the bottom skirt removed. Three $120^{\circ}$ tongues were developed and the right and non-coronary tongues were sutured to their respective sinuses. The tongue for the normal left sinus was removed and the proximal root anastomosis was carried across the top of the left sinus. The single right coronary ostium was implanted into the graft and the distal aortic anastomosis was completed.

\section{Completion}

Post-bypass, the leaflets exhibited excellent effective height and moved well with no residual leak (Figure 1). After valve repair, the mean valve gradient was $7 \mathrm{mmHg}$.

\section{Comments}

\section{Clinical results}

In the original clinical trials of aortic ring annuloplasty in aneurysm patients 7 years ago, outcomes were good (4), despite being very early in the technical learning curve. Shown in Figure 2, survival free of all complications was excellent, AI reduction was clinically and statistically significant, gradients were low and symptom reduction was good (4). Problems identified in the trials, such as annular suture untying and leaflet lacerations from long annular suture tails have been virtually eliminated in the subsequent 400 patients by lateral suture fixation. Thus, future clinical outcomes are likely to be even better.

\section{Advantages}

Geometric ring annuloplasty is a simple and effective technique for aortic valve repair during aortic aneurysm surgery (4). Important technical points include: (I) downsizing ring size whenever a question exists, (II) positioning ring posts low in the subcommissural triangles and (III) applying "lateral suture fixation" to every annular suture. With aortic root involvement, selective replacement of only the abnormal sinuses simplifies the procedure, using a remodeling root graft (5). 


\section{Caveats}

The most pathologic aortopathies, such as Ehlers-Danlos syndrome and Loeys-Dietz syndrome, might be excepted from this approach, and certainly, clinical judgment should be operative in all cases. Finally, more long-term followup data will be required for better understanding of this subject.

\section{Acknowledgments}

Funding: The research was supported by BioStable Science and Engineering (BSE), Austin Texas, USA (www.biostables-e.com).

\section{Footnote}

Conflicts of Interest: Drs. JS Rankin, MW Gerdisch, MJ Jasinski, D Mazzitelli, YH Choi, T Fischlein, A Weber, M Glauber, MS Si, GT Stavridis, and LM Wei are consultants for BSE. The other authors have no conflicts of interest to declare.

Cite this article as: Stavridis GT, Downey RS, Gerdisch MW, Hughes GC, Jasinski MJ, Rankin JS, Mazzitelli D, Aicher D, Fischlein T, Choi YH, Weber A, Friedrich I, Glauber M, Pfeiffer S, Miceli A, Si MS, Klokocovnik T, Wei LM, Deja MA, Badhwar V. Aortic valve repair for tri-leaflet aortic insufficiency associated with asymmetric aortic root aneurysms. Ann Cardiothorac Surg 2019;8(3):426-429. doi: 10.21037/ acs.2019.04.10

\section{References}

1. Rankin JS, Bone MC, Fries PM, et al. A refined hemispheric model of normal human aortic valve and root geometry. J Thorac Cardiovasc Surg 2013;146:1038.e1.

2. Crooke PS, Beavan LA, Griffin CD, et al. Design characteristics of a three-dimensional geometric aortic valve annuloplasty ring. Innovations (Phila) 2013;8:364-70.

3. Crooke PS, Beavan LA, Griffin CD, et al. A geometric model of the normal human aortic root and design of a fully anatomic aortic root graft. Innovations (Phila) 2015;10:57-62.

4. Rankin JS, Mazzitelli D, Fischlein T, et al. Geometric Ring Annuloplasty for Aortic Valve Repair During Aortic Aneurysm Surgery: Two-Year Clinical Trial Results. Innovations (Phila) 2018;13:248-53.

5. Urbanski PP, Jankulowski A, Morka A, et al. Patienttailored aortic root repair in adult marfanoid patients: Surgical considerations and outcomes. J Thorac Cardiovasc Surg 2018;155:43-51.e1. 